\title{
Antonovsky's Sense of Coherence Scale: Cultural Validation of Soc Questionnaire and Socio-Demographic Patterns in an Italian Population
}

\author{
Claudia Sardu ${ }^{1}$, Alessandra Mereu ${ }^{1}{ }^{*}$, Alessandra Sotgiu ${ }^{1}$, Laura Andrissi ${ }^{1}$, Maria Katarina Jacob- \\ son $^{2}$, Paolo Contu ${ }^{1}$ \\ ${ }^{I}$ Dipartimento di Sanità Pubblica, Università degli Studi di Cagliari, Monserrato (CA), Italy \\ ${ }^{2}$ Azienda Ospedaliero Universitaria di Cagliari, Monserrato(CA), Italy
}

\begin{abstract}
Background: The theory of salutogenesis entails that the ability to use resources for one's wellbeing is more important than the resources themselves. This ability is referred to as the Sense of Coherence (SOC). This paper present the cross-culturally adapted version of the Italian questionnaire (13 items), and the psychometric and statistical testing of the SOC properties. It offers for the first time a view of the distribution of SOC in an Italian sample, and uses a multivariate method to clarify the effects of socio-demographic determinants on SOC.

Methods: The cross-cultural adaptation of the English SOC questionnaire was carried out according to the guidelines reported in literature. To evaluate the psychometric and statistical properties we assessed reliability, validity and frequency distribution of the collected data. A Generalised Linear Model was used to analyse the effects of socio demographic variables on SOC.

Results: The Italian SOC scale demonstrates a good internal consistency $(\alpha=0.825)$. The model obtained with factorial analysis is not related to the traditional dimensions of SOC represented in more than one factor. The multivariate analysis highlights the joint influence of gender, age and education on SOC.

Conclusion: The validated Italian questionnaire is now available. Socio-demographic variables should be taken into account as confounders when SOC values among different populations are compared. Presenting data on SOC of the Italian population makes a control population available for comparisons with specific subgroups, such as patient populations. Now, the Italian challenge is to integrate the salutogenic approach into Public Health police.
\end{abstract}

Keywords: Sense of coherence, cross-cultural validation, multivariate analysis.

\section{INTRODUCTION}

The salutogenesis theory was introduced in 1970s by Antonovsky, a medical sociologist interested in stress theory, who gave a fundamental contribution to health research. He stated that diseases and stress occur everywhere and all the time and the question that came in his mind was how we can survive in spite of all this. Under the influence of stressors one comes under tension thereafter either succumbs under the stressors leading to a breakdown in a pathogenic direction or overcomes the strain and move towards the health, i.e. move in the salutogenic direction. The salutogenic model of health explains why people in stressful situation stay well and even are able to improve their health [1].

Health promotion and salutogenesis share a common vision for the wellbeing of the individual. Both theories recognise the ability to manage a number of positive or negative factors as good for individual health, in an everyday context [1].

According to the Ottawa Charter "To reach a state of complete physical, mental and social well-being, an

*Address correspondence to this author at the Dipartimento di Sanità Pubblica Università di Cagliari, SS $554(\mathrm{~km} \mathrm{4.500)} 09042$ Monserrato (CA), Italy; Tel/Fax: +39 070 6754658; E-mail: epidemiologia.unica@gmail.com individual or group must be able to identify and to realise aspirations, to satisfy needs, and to change or cope with the environment." "Health is therefore seen as a resource for everyday life, not the objective of living. Health is a positive concept emphasising social and personal resources, as well as physical capacities" [2].

Analogously, the theory of salutogenesis entails that the ability to use resources such as finances resources, knowledge, experience, self-esteem, healthy lifestyles, culture, social support for one's wellbeing is more important than the resources themselves. This ability is referred to as the Sense of Coherence (SOC); it can be defined as a way of seeing life and the ability to successfully manage the many stressors that are encountered in the course of life itself [3-9]. The SOC is a resource that enables people to manage tension, to reflect about their external and internal resources, to identify and mobilise those resources, to promote effective problem solving, and to resolve tension in a health-promoting manner [4]. It has three components: the cognitive component of comprehensibility (C) which refers to the extent to which one perceive the stimuli that confront one as consistent structured and clear; the instrumental or behavioural component of manageability (Ma) which is the extent to which one perceives that the resources at one's disposal are adequate to meet the lives demands; and the motivational component of meaningfulness (Me) which refers to the extent to which one 
feels that life makes sense emotionally. People should understand their lives, be understood by others, perceive that they are able to manage situation and most importantly, perceive that life is meaningful enough to find the motivation to continue [1].

Both Health Promotion and Salutogenesis emphasise the same core values: equity, participation and empowerment [1].

Antonovsky's theory of salutogenesis has become a central issue to the global development of health promotion in recent years. Since 2007, one of the four IUHPE (International Union for Health promotion and Education) global working groups dealing with issues central to the global health promotion development has been dedicated to Salutogenesis. This group explores the relevance of the salutogenic approach and framework for health promotion research and practice. Specifically, it aims to stimulate salutogenic research and development, to collect and analyse global research and practice evidence, to train and network professionals and researchers [10]. The evidence proves that the salutogenic model is a health-promoting resource that improves resilience and develops a positive subjective state of physical and mental health, quality of life and wellbeing [1114].

Health promoters are thus called on to enhance and develop knowledge about salutogenesis, to implement its principles and perspectives in all public health policy making, which includes incorporating the SOC among health indicators and using SOC in interventions, treatments, and school development [1]. The Folkhälsan Research Centre, which coordinates the Working Group on Global IUHPE Salutogenesis, has developed a web research database to produce continuous updates on systematic analysis for the global research on Salutogenesis [15].

The SOC questionnaire is the instruments for measuring Sense of Coherence at the individual level. It has been used in many general population studies, in groups of patients affected by different illnesses and in many public health sectors [14,16-18]. To date, it has been translated into at least 33 different languages in Europe and abroad and has been used in 32 countries (European countries, South Africa, Thailand, China and Japan). A systematic review of Salutogenesis research detected 325 surveys using the SOC questionnaire, but only five of them are from Latin countries ( 2 from France, 2 from Brasil, 1 from Colombia) [4]. Therefore, the translation and cultural validation into Italian of the shortform (13 items) of Antonowsky's Sense of Coherence Scale (SOC) can make a valuable contribution to salutogenic research and development. Additionally, the systematic review of the current literature on salutogenesis points out contrasting results about the factorial structure of the three dimensions of the SOC (comprehensibility, manageability, and meaningfulness) and the need of a thorough statistical analyses [4].

The aim of this paper is to present the cross-culturally adapted version of the Italian questionnaire, and the psychometric and statistical testing of the SOC properties. Moreover this paper offers for the first time a view of the distribution of SOC score in a sample of the Italian popula- tion. To our knowledge, this is the first report that uses a multivariate method to clarify the effects of sociodemographic determinants on SOC score evaluated by the 13 items questionnaire.

\section{METHODS}

\section{Cross Cultural Adaptation Process}

The cross-cultural adaptation process of the English version of the SOC questionnaire was carried out according to the guidelines reported in literature [19].

In the first step, the questionnaire was translated independently by four native-speaking Italian translators with experience in Health Promotion. The translators performed their own translations individually, each noting problems and difficulties. In the second step, they met together to produce a common draft of the Italian version of the SOC questionnaire.

This version was then translated back into English by a professional, native-speaking English translator, who had not participated in the earlier stage and had never read the original version of the questionnaire in English. Consensus on the preliminary Italian version was obtained after a meeting with expert translators in the Health Promotion field.

The preliminary questionnaire was pre-tested on a group of 100 volunteers, comprised of men and women of 20 years of age or more and with different educational levels. The volunteers were asked to fill in the questionnaire and to discuss the comprehensibility and clearness of the questions. Later, the Italian version of SOC was slightly modified according to the suggestions and queries from the volunteers, producing the final Italian version of the SOC questionnaire. This version was submitted to the Folkhälsan Research Centre, which is carrying out an ongoing systematic analysis of salutogenic research.

\section{Testing Of The Final Italian Version of The Soc Questionnaire}

The final Italian version of the SOC questionnaire was tested in Sardinia, Italy, in 2010. A representative sample of the regional territory was selected from demographic data through quota sampling; sample numbers were selected according to gender, age, education (low level = primary school, high level $=$ high degree or higher), and area of residence (urban and rural). The refusal rate was $<1 \%$. The total sample population consisted of 913 citizens (449 male and 464 female): 380 living in urban areas and 533 living in rural areas, all between 20 and 85 years of age.

The questionnaire was administered by 18 interviewers trained by the researchers of the Department of Public Health of University of Cagliari, Italy.

\section{Statistical Analysis}

To evaluate the psychometric and statistical properties of the Italian version of the SOC scale, we assessed reliability, validity and frequency distribution of the collected data.

To test the hypothesis of a Gaussian distribution of SOC score, the SOC values associated to percentiles were compared with the corresponding expected SOC values based on the mean $(\mu)$ and standard deviation $(\sigma)$. Thus, the $2.5^{\text {th }}$ per- 
Table1. Comparison Between SOC Values Associated with Percentiles and those Estimated Based on the Mean and Standard Deviation

\begin{tabular}{|c|c|c|c|}
\hline Percentiles & Soc Values Associated with Percentiles & Soc Values Estimated by Mean and sd & Gaussian Distribution \\
\hline \hline $2,5^{\text {th }}$ & 32.3 & 34.5 & $\mu-2 \sigma$ \\
\hline $16^{\text {th }}$ & 48.0 & 47.1 & $\mu-\sigma$ \\
\hline $50^{\text {th }}$ & 60.0 & 60.3 & $\mu$ \\
\hline $84^{\text {th }}$ & 74.0 & 73.6 & $\mu+\sigma$ \\
\hline $97,5^{\text {th }}$ & 85.0 & 86.2 & $\mu+2 \sigma$ \\
\hline
\end{tabular}

centile was compared to the $\mu-2 \sigma$ value, the $16^{\text {th }}$ percentile to the $\mu-\sigma$ value, the $50^{\text {th }}$ to the $\mu$ value, the $84^{\text {th }}$ to the $\mu+\sigma$ value, and the $97.5^{\text {th }}$ to the $\mu+2 \sigma$. If the values associated with the percentiles overlapped with those expected, then a normal distribution is a good representation of the actual population. Consequently, the mean and standard deviation adequately describe this population [20]. The normality of the data distribution was also investigated using the Kolmogorov-Smirnov test.

The reliability was tested measuring the internal consistency through Cronbach's $\alpha$ coefficient.

The analysis of construct validity was carried out through a factor analysis of the relationships among the items of the SOC questionnaire. The assumption of the model is that the relationships may be explained by a few unobserved variables, which are called factors. Only factors with eigenvalues greater than 1.0 were extracted. The Quartimax method was used for the rotation procedure. For each item, a communality value was calculated, that is, the amount of information that is kept by the factorial components and the item's correlation with each of the found components. The higher the communality, the more the model is able to represent the global information contained in the data. The more single items are correlated with only one component, the more the model is able to highlight split dimensions.

Finally the distribution of SOC score was analysed according to area of residence (urban vs. rural), gender (men vs. women), age ( $<30$ years of age vs. 30-59 years of age, and $\geq 60$ years of age vs. 30-59 years of age) and educational level (high=senior high school or more vs. low=junior high school or less). A Generalised Linear Model was used to test null hypotheses about the effects of other variables on the means of various groupings of a single dependent variable $[21,22]$. The GLM procedure provides regression analysis and analysis of variance for one dependent variable by one or more independent variables. The GLM was carried out through a backward stepwise procedure, starting with the full model, including independent variables and second order interactions between them, and deleting step by step nonsignificant interactions and variables. The strength of the association of each independent variable with the dependent variable (after the effects of all other independent variables was accounted for) was expressed as Partial Eta Squared.

\section{RESULTS}

In the process of translation from English into Italian, the only term translated differently by the four translators was the ward "feelings". In English, the word "feeling" means both "something that you feel through the mind or through the senses like hunger, sadness" and "the emotions of a person". In Italian, the two concepts are expressed, respectively, with the words "sensazione" and "emozione". Consensus on the choice of the term "sensazione" was reached after consultation with the researchers at the Folkhälsan Research Centre.

The translation of the question "Many people - those with even a strong character - sometimes feel like sad sacks (losers) in certain situations. How often have you felt this way in the past?" revealed a minor difficulty of idiomatic equivalence. In the Italian version, it was necessary to eliminate the idiom "sad sacks" for which there is no corresponding expression in our language. The significance of the question is still guaranteed by the translation of the word "losers".

The back-translation of the questionnaire overlaps the original English version; thus, the Italian translation was considered appropriate. The questionnaire is now available on the web-site http:www.salutogenesis.fi.

The analysis of the frequency distribution of SOC score showed that values associated with percentiles were substantially equal to those estimated on the basis of mean and standard deviation (Table 1). This outcome was also confirmed by the Kolmogorov Smirnov test which showed a $p$ value of 0.20 . According to these results, the Gaussian distribution is a good representation of SOC score.

Consequently, the mean and standard deviation adequately describe this population.

In the Sardinian population, the mean SOC value is 60.3 (95\% Confidence Interval 59.1-61.8), and the standard deviation is 13.6 .

Comprehensibility of the questionnaire was confirmed by the high number of questionnaires completed in full, equal to 904 of 913 . Only nine questionnaires had a question left blank, but never the same question.

A Cronbach's $\alpha$ of 0.825 demonstrated a good internal consistency of the Italian version of the SOC scale.

The factorial analysis resulted in three factors with an eigenvalue $>1$ and accounted for $52 \%$ of the variance (Table 2). The communalities range from 0.267 to 0.766 , highlighting a not fully satisfactory representation of some items in the model. After rotation with the Quartimax method, the first component, accounting for $28.1 \%$ of the explained variance, embraced 7 items from the SOC scale. The second component, accounting for $12.3 \%$ of the explained variance, embraced 2 items. The third component, $12.3 \%$ of the explained variance, embraced two items. The items "Many people sometimes feel like sad sacks (losers) in certain situa- 
Table 2. Factorial Analysis Rotated Component Matrix (Quartimax)

\begin{tabular}{|c|c|c|c|c|c|}
\hline $\begin{array}{l}\text { Antonovsky's } \\
\text { Components of } \\
\text { Soc }\end{array}$ & Communalities & Items & \multicolumn{3}{|c|}{ Components } \\
\hline $\mathrm{C}$ & 0,476 & Do you have the feeling that you are in an unfamiliar situation and don't know what to do? & 0,687 & 0,027 & 0,062 \\
\hline $\mathrm{C}$ & 0,587 & Do you have very mixed-up feelings and ideas? & 0,686 & 0,340 & 0,028 \\
\hline Ma & 0,517 & How often do you have feelings that you're not sure you can keep under control? & 0,671 & 0,258 & $-0,016$ \\
\hline $\mathrm{C}$ & 0,481 & Does it happen that you have feelings inside you would rather not feel? & 0,637 & 0,195 & 0,192 \\
\hline $\mathrm{Me}$ & 0,372 & Do you have the feeling that you don't really care about what goes on around you? & 0,585 & $-0,168$ & $-0,045$ \\
\hline $\mathrm{C}$ & 0,267 & $\begin{array}{l}\text { When something happened, have you generally found that: you overestimated or underes- } \\
\text { timate its importance - you saw things in the right proportion }\end{array}$ & 0,509 & $-0,069$ & $-0,052$ \\
\hline $\mathrm{Me}$ & 0,627 & Until now your life has had: no clear goals or purpose at all - very clear goals and purpose & 0,300 & $\mathbf{0 , 7 2 3}$ & 0,116 \\
\hline $\mathrm{Me}$ & 0,598 & $\begin{array}{l}\begin{array}{l}\text { Doing the things you do every day is: a source of deep pleasure and satisfaction - a source } \\
\text { of pain and boredom }\end{array}\end{array}$ & 0,309 & 0,709 & $-0,018$ \\
\hline $\mathrm{Me}$ & 0,560 & $\begin{array}{l}\text { How often do you have the feeling that there's little meaning in the things you do in your } \\
\text { daily life? }\end{array}$ & 0,599 & 0,434 & $-0,115$ \\
\hline Ma & 0,355 & $\begin{array}{l}\text { Many people sometimes feel like sad sacks (losers) in certain situations. How often have } \\
\text { you felt this way in the past? }\end{array}$ & 0,469 & 0,308 & 0,200 \\
\hline
\end{tabular}

Table 3. Mean SOC Values According to Gender, Education and Age Category

\begin{tabular}{|c|c|c|c|}
\hline Gender & Education & Age category & Mean SOC \\
\hline \multirow{4}{*}{ Women } & \multirow{2}{*}{ Low } & $<30$ & 53,7 \\
\hline & & $\geq 30$ & 57,2 \\
\hline & \multirow{2}{*}{ High } & $<30$ & 56,8 \\
\hline & & $\geq 30$ & 61,3 \\
\hline \multirow{4}{*}{ Men } & \multirow{2}{*}{ Low } & $<30$ & 56,2 \\
\hline & & $\geq 30$ & 60,7 \\
\hline & \multirow{2}{*}{ High } & $<30$ & 56,9 \\
\hline & & $\geq 30$ & 66,8 \\
\hline
\end{tabular}

tions. How often have you felt this way in the past?" and "How often do you have the feeling that there's little meaning in the things you do in your daily life?" showed correlation with the first, second and third components; thus, they cannot be assigned to any component.

The internal consistency calculated for each component was good for the first $(\alpha=0.798)$ and the third components ( $\alpha=0.738$ ), while it was less satisfactory for the second component $(\alpha=0.578)$.

The Generalised Linear Model did not highlight any interaction between socio-demographic variables and revealed that gender, age and education have a slight effect on SOC, while area of residence does not influence SOC level.

The final model had an $\mathrm{R}^{2}$ value of 0.072 , indicating that only $7.2 \%$ of the variance in the SOC score was accounted for by gender, age and education. Gender (men vs. women) was significantly associated with $\operatorname{SOC}(F=21.2 ; p<0.001)$, with a Partial Eta Squared of 0.023. Education level (high vs. low) was significant associated with $\operatorname{SOC}(F=28,3 ; p<$ 0.001), with a Partial Eta Squared of 0.031. Age was also significantly associated with SOC $(F=25.6 ; p<0.001)$, with the young group ( $<30$ years of age) significantly different from both the 30 to 59 and $\geq 60$ groups, while the 30 to 59 and $\geq 60$ groups were not significantly different from each other.

The SOC mean values in the total sample and according to gender, education and age are reported in Table $\mathbf{3}$. The lowest mean value of SOC was observed in women with low education and under 30 years of age (53.7); a slightly higher SOC mean value was detected in the $\geq 30$ age group (57.2). Among women with high education, an increase of the mean SOC value was observed from the youngest age class (56.8) to the oldest (61.3).

Among men with low education, the mean SOC values ranged from 56.2 in the young group to 60.7 in the $\geq 30$ age group; these results are similar to values observed in women with high education.

The highest SOC mean values are detected in men with high education, where a pronounced increase across age classes is evident: the mean SOC value ranges from 56.9 in the young group to 66.8 in the $\geq 30$ age group. 


\section{DISCUSSION}

This paper presents the cross-culturally adapted version of the Italian questionnaire on Sense of Coherence, with psychometric and statistical testing of SOC properties. The translation did not present any major difficulty. The validated Italian questionnaire is now available on the official web-site created by the Folkhälsan Research Centre, which is the coordinator of the Working Group on Global IUHPE Salutogenesis [15].

Current literature emphasises the importance of implementing the knowledge and salutogenesis SOC questionnaire, given that they have important implications for public health. "The salutogenic approach creates populations that live longer, are more prone to choose positive behaviour, have less problems with illness (acute or chronic), have shorter and less frequent needs to use the health care system and they endure stress better. Further, these populations perceive they are in a better health, enjoy a good quality of life and are mentally well" [11]. The Italian Public Health System considers disease treatment a priority, which is guaranteed by providing health care free of charge to Italian citizens and immigrants, both regular and irregular. A health care system of this type hardly supports increasingly high costs and fails to fully satisfy the aspirations of people's health. People are increasingly aware that to be healthy and have a good quality of life, it is not enough to be treated, but it is necessary to have the opportunity to pursue their aspirations, to satisfy needs, and to change or cope with the environment $[1,2]$. Reorienting Public Health towards a salutogenic approach could improve quality of life and, at the same time, could help to keep health costs under control [14, 23]. The availability of the official Italian version of the SOC questionnaire lays the foundations to initiate the development of research on salutogenesis to give new input into public health. Currently, in Latin countries, the research on salutogenesis is in its infancy [4]. The Italian version of the questionnaire, available on the salutogenesis web-site, is the first example of an official translation into the new-Latin language and can be a useful reference for other countries in the Mediterranean and Ibero-American areas.

Moreover, this paper offers for the first time a view of the distribution of SOC score in a sample of the Sardinian population, evaluating the psychometric and statistical properties of the Italian version of the SOC questionnaire.

The Italian version of the SOC scale demonstrates a good internal consistency $(\alpha=0.825)$, agreeing with the current literature $[4,24]$.

With regard to construct validity, the model obtained with factorial analysis, although three-dimensional and with a good explained variance, is not related to the traditional dimensions of SOC represented in more than one factor. This is in agreement with previous research on SOC factorial dimension that showed contrasting results normally not fitting with the dimensions of comprehensibility, manageability, and meaningfulness [25, 26].

Our results are analogous to those reported by Larsson and Kallenberg ("Dimensional Analysis of Sense of Coherence Using Structural Equation Modelling") in Sweden [26].
In their model, two dimensions overlap with our second and third ones, both including two items. Five other items are assigned to the first dimension in both models, although in ours, a sixth and seventh are included. Two items in our model and four in the Swedish appear to not assignable to the three dimensions.

Our research confirms that the SOC scores follow a Gaussian distribution. The SOC mean values observed in the Sardinian population are homogeneous between urban and rural areas and are not different from the mean values reported in other European countries, thus confirming that SOC seems to be independent of cultural context $[4,5]$.

Previous studies report conflicting results about the effect of socio-demographic determinants on SOC, highlighting the need for further studies $[12,13,27,28,29]$. Such discrepancies may be due to the fact that data are analysed through a univariate method, without taking into account potential confounding effects, and with a low power to detect weak associations. Our study is based on multivariate method that highlights the influence of gender, age and education on SOC. Therefore, these variables should be taken into account as confounders when SOC values among different populations are compared.

The similarity between metropolitan and rural area in mean SOC levels suggests that our data can be generalised at the national level. Presenting data on SOC of the Italian general population makes a control population available for future comparisons with specific subgroups, such as patient populations. Now, the Italian challenge is to integrate the salutogenic approach into Public Health police.

Competing interests: none declared.

\section{WHAT IS ALREADY KNOWN}

- Antonovsky's theory of Salutogenesis has become a central issue to the global development of health promotion. Since 2007, one of the IUHPE global working groups dealing with issues central to the global health promotion development has been dedicated to Salutogenesis. The evidence proves that the salutogenic model is a health-promoting resource that improves quality of life and wellbeing.

- A systematic review of Salutogenesis detected 325 surveys using the SOC questionnaire, but only five of them are from Latin countries, and none is from Italy.

- Current literature shows conflicting results about the effect of socio-demographic determinants on SOC; these results are based on data analysis performed through univariate method.

\section{WHAT YOUR PAPER ADDS}

- The validated version of the Italian questionnaire on Sense of Coherence (13 items).

- This paper offers for the first time a descriptive analysis of SOC in a sample of the Italian population.

- The use of the multivariate method highlights the joint influence of gender, age and education on SOC. 


\section{CONFLICT OF INTEREST}

None declared.

\section{ACKNOWLEDGEMENT}

None declared.

\section{REFERENCE}

[1] Eriksson M, Lindström B. A Salutogenic interpretation of Ottawa Charter. Health Promot Int 2008; 23: 190-9.

[2] The Ottawa Charter for Health Promotion. WHO 1986

[3] Lindström B, Eriksson M. Salutogenesis. J Epidemiol Community Health 2005; 59: 440-2

[4] Eriksson M. Unravelling the mistery of Salutogenesis. FOLKHÄLSAN RESEARCH CENTRE HEALTH PROMOTION RESEARCH PROGRAMME. Helsinky: Turku, 2007.

[5] Eriksson M, Lindström B. Validity of antonovsky's sense of coherence scale: a systematic review. J Epidemiol Community Health 2005; 59: 460-6.

[6] Eriksson M, Lindström B. Antonovsky's sense of coherence scale and its relation with quality of life: a systematic review. J Epidemiol Community Health 2007; 61: 938-44.

[7] Antonovsky A. The structure and properties of the sense of coherence scale. Soc Sci Med 1993; 36: 725-33.

[8] Antonovsky A. The salutogenic model as a theory to guide health promotion. Health Prom Int 1996; 11: 11-8.

[9] Antonovsky A. The sense of coherence: An historical and future perspective. Israeli J Med Sci 1996; 32: 170-8.

[10] IUHPE (International Union for Health Promotion and Education). Global Working Group on Salutogenesis. http://www.iuhpe.org/?page $=494 \&$ lang $=$ en.

[11] Lindström B, Eriksson M. The Hitchhiker's guide to Salutogenesis. Helsinki, FOLKHÄLSAN, 2010.

[12] Wainwright NW, Surtees PG, Welch AA, et al. Healthy lifestyle choices: could sense of coherence aid health promotion? J Epidemiol Community Health 2007; 61: 871-6.

[13] Poppius E, Virkkunen H, Hakama M, et al. The sense of coherence and risk of injuries: role of alcohol consumption and occupation. $\mathbf{J}$ Epidemiol Community Health 2008; 62: 35-41.

[14] Lindström B, Eriksson M. Contextualizing salutogenesis and Antonovsky in public health development. Health Promot Int 2006; 21: $238-44$.
[15] FOLKHÄLSAN - Salutogenesis. Available at: http://www. salutogenesis.fi.

[16] Bergha H, Baigia A, Fridlund B, et al. Life events, social support and sense of coherence among frequent attenders in primary health care. Public Health 2006; 120: 229-36.

[17] Kouvonen AM, Väänänen Ari, Woods SA, et al. Sense of coherence and diabetes: A prospective occupational cohort study. BMC Public Health 2008; 8: 46.

[18] Richardson CG, Ratner PA. Sense of coherence as a moderator of the effects of stressful life events on health. J Epidemiol Community Health 2005; 59: 979-84.

[19] Beaton DE, Bombardier C, Guillemin F, et al. Guidelines for the process of cross-cultural adaptation of self-report measures. Spine (Phila Pa 1976) 2000; 25: 3186-91.

[20] Glanz AS. Statistica per discipline biomediche. Milano: McGrawIll 1997:16-9.

[21] Neter J, Wasserman W, Kutner MH. Applied linear regression models. USA, Richard D. Irvin, INC 1989: 225-308.

[22] Kleinbaum DG, Kupper LL, Muller KE. Applied Regression Analysis and other Multivariable Methods. Boston, PWS-KENT Publishing Company 1988: 102-16.

[23] Becker CM, Glascoff MA, Felts WM. Salutogenesis 30 Years Later: Where do we go from here? Int Electron J Health Educ 2010; 3: 25-32.

[24] Olsson M, Gassne J, Hansson K. Do different scales measure the same construct? three sense of coherence scales. J Epidemiol Community Health 2009; 63: 166-7.

[25] Togari T, Yamazaki Y, Nakayama K, et al. Construct validity of Antonovsky's sense of coherence scale: Stability of factor structure and predictive validity with regard to the well-being of Japanese undergraduate students from two-year follow-up data. JSHHE 2008; 74: 71-86.

[26] Larsson G, Kallenberg K. Dimensional analysis of sense of coherence using structural equation modelling. Eur J Pers 1999; 13: 5161.

[27] Lundberg O, Nystrom Peck M. Sense of Coherence, social structure and health. Eur J Public Health 1994; 4: 252-7.

[28] Mahammadzadeha A, Poursharifib H, Alipoura A. Validation of sense of coherence (SOC) 13-item scale in iranian sample. Procedia Soc Behav Sci 2010; 5: 1451-5.

[29] Larsson G, Kallenberg K. Sense of coherence, socioeconomic conditions and health. Eur J Public Health 1996; 6: 175-80.

Received: April 20, 2011

Revised: November 28, 2011

Accepted: November 29, 2011

(C) Sardu et al.; Licensee Bentham Open.

This is an open access article licensed under the terms of the Creative Commons Attribution Non-Commercial License (http://creativecommons.org/licenses/by-nc/3.0/) which permits unrestricted, non-commercial use, distribution and reproduction in any medium, provided the work is properly cited. 